\title{
AVANCES Y ANÁLISIS EN LA CONSTRUCCIÓN DE INDICADORES DE PRESIÓN, ESTADO Y RESPUESTA PARA LA CALIDAD AMBIENTAL EN EL PERIURBANO DE VILLA MARÍA, CÓRDOBA, ARGENTINA.
}

\section{ADVANCES AND ANALYSIS IN THE CONSTRUCTION OF PRESSURE, STATE AND RESPONSE INDICATORS FOR ENVIRONMENTAL QUALITY IN THE PERIURBANO OF VILLA MARÍA, CÓRDOBA, ARGENTINA.}

http://dx.doi.org/10.30972/geo.15292905

\section{Ing. en Ecología Guzmán Leticia Ana}

Becaría doctoral del Centro de Investigación y Transferencia (CIT Villa María- CONICET). Docente de la Licenciatura en Ambiente y Energías Renovables (LAER) del Instituto Académico y Pedagógico de Ciencias Básicas y Aplicadas (IAPCBA), Universidad Nacional de Villa María (UNVM).

E mail: proy.inv.ia@gmail.com

\section{Dra. en Ciencias Geología Becker Analía Rosa}

Docente e investigadora de la Facultad de Ciencias Exactas, Físico-Química y Naturales de la Universidad Nacional de Río Cuarto (UNRC) y

Licenciatura en Ambiente y Energías Renovables (LAER) del Instituto Académico y

Pedagógico de Ciencias Básicas y Aplicadas (IAPCBA), Universidad Nacional de Villa María (UNVM).

\section{Dr. en Ciencias Biología José Camilo Bedano}

Docente e Investigador (CONICET) Facultad de Ciencias Exactas, Físico-Química y

Naturales de la Universidad Nacional de Río Cuarto (UNRC).

\section{RESUMEN}

Desde hace décadas las organizaciones gubernamentales y no gubernamentales han aportado al desarrollo del estudio de la calidad ambiental. Así mismo, se vienen desarrollando estudios en las áreas rurales, urbanas, naturales y periurbano.

En el periurbano se despliegan complejos fenómenos ecológicos y sociales, con una gran dinámica de transformación. Así mismo, existe una dependencia por parte de las ciudades a las actividades que allí se desarrollan.

El periurbano de Villa María se analizó desde la ecología del paisaje y se construyeron parches de uso del suelo como forma de análisis espacial. Se adaptaron y crearon indicadores propios para la localidad.

Los indicadores establecidos pueden medir la presión, el estado y respuesta que se genera en el territorio periurbano. El ambiente es un sistema complejo que debe ser contemplada en los estudios, donde las técnicas de medición sean sencillas y se constituyan en herramientas de gestión.

PALABRAS CLAVES: producción; parches; uso del suelo.

Publicado en formato digital: Ing. L. A. Guzmán, Dra. A Becker y Dr. J.C. Bedano. AVANCES Y ANÁLISIS EN LA CONSTRUCCIÓN DE INDICADORES DE PRESIÓN, ESTADO Y RESPUESTA PARA LA CALIDAD AMBIENTAL EN EL PERIURBANO DE VILLA MARÍA, CÓRDOBA, ARGENTINA. Revista Geográfica Digital. IGUNNE. Facultad de Humanidades. UNNE. Año 15. № 29. Enero - Junio 2018. ISSN 1668-5180 Resistencia, Chaco.

En: http://hum.unne.edu.ar/revistas/geoweb/default.htm - http://dx.doi.org/10.30972/geo.15292905 
Revista Geográfica Digital. IGUNNE. Facultad de Humanidades. UNNE. Año 15. № 29.

Enero - Junio 2018. ISSN 1668-5180 Resistencia, Chaco

\begin{abstract}
For several decades researchers from governmental and non-governmental organizations have contributed to the development of the study of environmental quality. Also, several efforts were made to study the rural, urban, natural and periurban areas.

In the periurban complex unfold ecological and social factors, with a great dynamic of transformation. Likewise, there is a dependence on the part of the cities to the activities that are developed in these territories.

The periurbano of Villa Maria was analyzed from ecology of the landscape and it was built patches of land use as a form of spatial analysis. They adapted and created their own indicators for the locality.

The established indicators can measure the pressure, the state and the response that is generated in the periurban territory. The environment is a complex system that must be contemplated in studies, where measurement techniques are simpel and constitute management tools.
\end{abstract}

Keywords: production; patches; land use.

\title{
Introducción
}

El ambiente es un sistema complejo compuesto por factores sociales, culturales, ambientales, naturales y físicos, que interactúan entre sí, determinando la forma, el carácter, el comportamiento y la supervivencia de ambos (Gómez Orea, 2013). Además, es sustento de las actividades humanas debido a que es fuente de recursos naturales que pueden ser aprovechados por la sociedad.

El periurbano es la zona de transición entre la ciudad y el campo donde se despliegan fenómenos tanto sociales como ecológicos (Crojethovich \& Barsky, 2012). Hay diferentes trabajos que establecen las particularidades de este espacio. En tal sentido, el área periurbana presenta características sociales y de producción heterogéneas en las cuales se encontran extracción de suelo (ladrilleras), producción hortícola, avicultura, ganadería, establecimientos industriales agrupados y/o dispersos, así como también, barrios cerrados (Di Pace \& Caride Bartrons, 2012).

Según el Plan Estratégico Territorial Nacional (2008) la provincia de Córdoba cuenta con una red urbana que cubre aproximadamente toda su superficie. Los principales núcleos urbanos son: Gran Córdoba (con Villa Carlos Paz), Villa María, San Francisco, Río Cuarto que acumulan el $64 \%$ de la población. Por otra parte, la Ciudad de Villa María cuenta con la mayor población del departamento General San Martín, del cual es cabecera, y es la tercera localidad más grande de la provincia con 80.006 habitantes censo 2010.

Por otra parte, Allen (2003) establece que los territorios municipales cuentan con una gran dinámica de transformación entre lo rural y lo urbano, que pueden ser abordados desde los enfoques ecológicos y socioeconómicos. En cuanto a lo ecológico, Allen (2003) centra su análisis desde una visión sistémica en la cual confluyen los subsistemas "naturales", "productivos" y "urbanos".

Para medir el camino hacia el desarrollo sustentable la Conferencia de las Naciones Unidades sobre el Ambiente y el Desarrollo - Río `92 (UNCED) marcó un hito muy especial al establecer la necesidad de desarrollar y aplicar diferentes metodologías para determinar el estado del ambiente y para monitorear los cambios ocurridos a nivel local, nacional, regional y global. La determinación de estos cambios podría ayudar a realizar una mejor evaluación de las dimensiones de los diferentes problemas ambientales, identificar y evaluar los resultados de la aplicación de las convenciones internacionales y los programas de acción, así como también, las políticas nacionales. A partir de Río '92 se desarrollo un uso generalizado de indicadores para la evaluación de la Calidad Ambiental, Sustentabilidad, Desarrollo Sustentable, Riesgo, Vulnerabilidad, Planificación Territorial, entre otros. Uno de los antecedentes más importante surge de la Organización para la Cooperación y el Desarrollo Económico (OECD, 2008) cuando publica el set preliminar de indicadores ambientales y

Publicado en formato digital: Ing. L. A. Guzmán, Dra. A Becker y Dr. J.C. Bedano. AVANCES Y ANÁLISIS EN LA CONSTRUCCIÓN DE INDICADORES DE PRESIÓN, ESTADO Y RESPUESTA PARA LA CALIDAD AMBIENTAL EN EL PERIURBANO DE VILLA MARÍA, CÓRDOBA, ARGENTINA. Revista Geográfica Digital. IGUNNE. Facultad de Humanidades. UNNE. Año 15. № 29. Enero - Junio 2018. ISSN 1668-5180 Resistencia, Chaco.

En: http://hum.unne.edu.ar/revistas/geoweb/default.htm 
Revista Geográfica Digital. IGUNNE. Facultad de Humanidades. UNNE. Año 15. № 29. Enero - Junio 2018. ISSN 1668-5180 Resistencia, Chaco

establece el modelo de Presión, Estado y Respuesta (modelo PER) para evaluar y monitorear las condiciones del ambiente a nivel nacional, regional y mundial. Este modelo parte de una relación de causalidad donde se considera que las actividades humanas ejercen una presión sobre el ambiente, cambiando su calidad y la calidad de los recursos naturales (el estado). La información sobre estos cambios enriquece las instancias de toma de decisiones en la sociedad (la respuesta). Los indicadores son herramientas que determinan el estado de los componentes físicos, ecológicos, sociales y geográficos del ambiente, que sirven de base para la comparación a través del tiempo (Altieri \& Nicholls, 2006).

Es así que el ambiente es el entorno vital; constituido por los elementos físicos, biológicos, económicos, sociales, culturales y estéticos que interactúan entre sí, con el individuo y la comunidad, determinando la forma, el carácter, el comportamiento y la supervivencia de ambos (Gómez Orea \& Villarino, 2013). En función de lo planteado previamente la calidad ambiental es entendida como el estado en el cual los componentes del ambiente se relacionan saludablemente sin generar un perjuicio ente ellos que lleve a un deterioro.

Por ello, los espacios periurbanos cuenta con la capacidad de trabajar como zonas de amortiguación ante las presiones que el sistema urbano ejerce sobre los recursos naturales, esta condición de amortiguación es parte del aporte en calidad (Hernández Jiménez et al, 2009) siempre y cuando los usos del suelo que se realicen en dicho periurbano no vayan en detrimento de los recursos naturales. En este trabajo se pretende evaluar diferentes indicadores de tipo PER (Presión, Estado y Respuesta) aplicados a la calidad ambiental en el periurbano de Villa María, Córdoba, Argentina en función de los parches de uso de suelo.

\section{Materiales y Métodos}

El estudio es en el área periurbana de la ciudad de Villa María, provincia de Córdoba, Argentina. La región se caracteriza por presentar un clima templado subhúmedo donde las temperaturas estivales son de $24^{\circ} \mathrm{C}$ e invernales de $9,6^{\circ} \mathrm{C}$ con una amplitud de $14^{\circ} \mathrm{C}$ y una precipitación anual de $780 \mathrm{~mm}$. Se observan variaciones estacionales de la precipitación, destacándose períodos de deficiencia prácticamente todo el año excepto en marzo, abril y mayo. Geomorfológicamente, Villa María se encuentra en el ámbito de la gran planicie Chaco-Pampeana, caracterizada por una extensa sedimentación continental en donde se reconocen restos de abanicos aluviales coalescentes muy disectados, compuestos por sedimentos fluviales que gradualmente se interdigitan con limos y arenas fluvio-eólicas y materiales loessoides (Carignano et al., 2014). En este complejo relieve fluvio-eólico, con problemáticas de salinidad y anegamiento, se desarrollan, predominantemente, molisoles (Haplustoles, Argiustoles, Natrustoles, Natralboles y Argialboles) y en menor proporción alfisoles (Natracualfes y Albacualfes) (Bosnero et al., 2006).

La vegetación natural del área corresponde a la Provincia Fitogeográfica del Espinal, Distrito del Algarrobo (Cabrera, 1971), con una fuerte pérdida de sus características fisonómicas por modificación de los usos del suelo. La actividad dominante es agrícola-ganadera, con un importante crecimiento de industrias agropecuarias en la zona. La modificación de la estructura ecológica, el uso del suelo y agua fueron variando con el tiempo, generando una mayor presión en el sistema (Seiler \& Vianco, 2014).

Se determinó el espacio periurbano y sus límites internos en función de las parcelas rurales establecidas por el municipio, la Ordenanza Municipal № 6401 y 6402 del 2012 de zonificación y el Censo Nacional de Hogares del 2010, el limite externo se estableció en función hasta donde llega la jurisdicción municipal (Figura 1). Posteriormente, se establecieron los parches de uso del suelo(1) (Figura 2) (Guzmán et al., 2016) y se analizó la metodología de Indicadores del modelo Presión, Estado y Respuesta (Cantú et al., 2008). La Presión son atributos que describen actividades o aspectos humanos que ejercen presión sobre el ambiente, esto es la causa subyacente de un problema (Por ejemplo: crecimiento de la población, cambio en el uso de la tierra). El Estado son atributos que generalmente describen algunas características físicas medibles del ambiente que son el resultado de las presiones (Por ejemplo: calidad del agua, calidad o deterioro del suelo). La Respuesta son atributos que informan en qué grado la

Publicado en formato digital: Ing. L. A. Guzmán, Dra. A Becker y Dr. J.C. Bedano. AVANCES Y ANÁLISIS EN LA CONSTRUCCIÓN DE INDICADORES DE PRESIÓN, ESTADO Y RESPUESTA PARA LA CALIDAD AMBIENTAL EN EL PERIURBANO DE VILLA MARÍA, CÓRDOBA, ARGENTINA. Revista Geográfica Digital. IGUNNE. Facultad de Humanidades. UNNE. Año 15. № 29. Enero - Junio 2018. ISSN 1668-5180 Resistencia, Chaco.

En: http://hum.unne.edu.ar/revistas/geoweb/default.htm 
sociedad está respondiendo a los cambios y la protección ambientales (Ejemplo: políticas, acciones). Se tomaron los antecedentes realizados por Cantú et al. (2008), Di Pace \& Caride Bartrons (2012), Zulaica (2010) y la metodología de Seiler \& Vianco (2014), sin embargo aquellos indicadores correspondientes a los servicios ambientales fueron formulados en función de los principios de la economía ecológica planteados por Martínez Alier (1998, 2004).

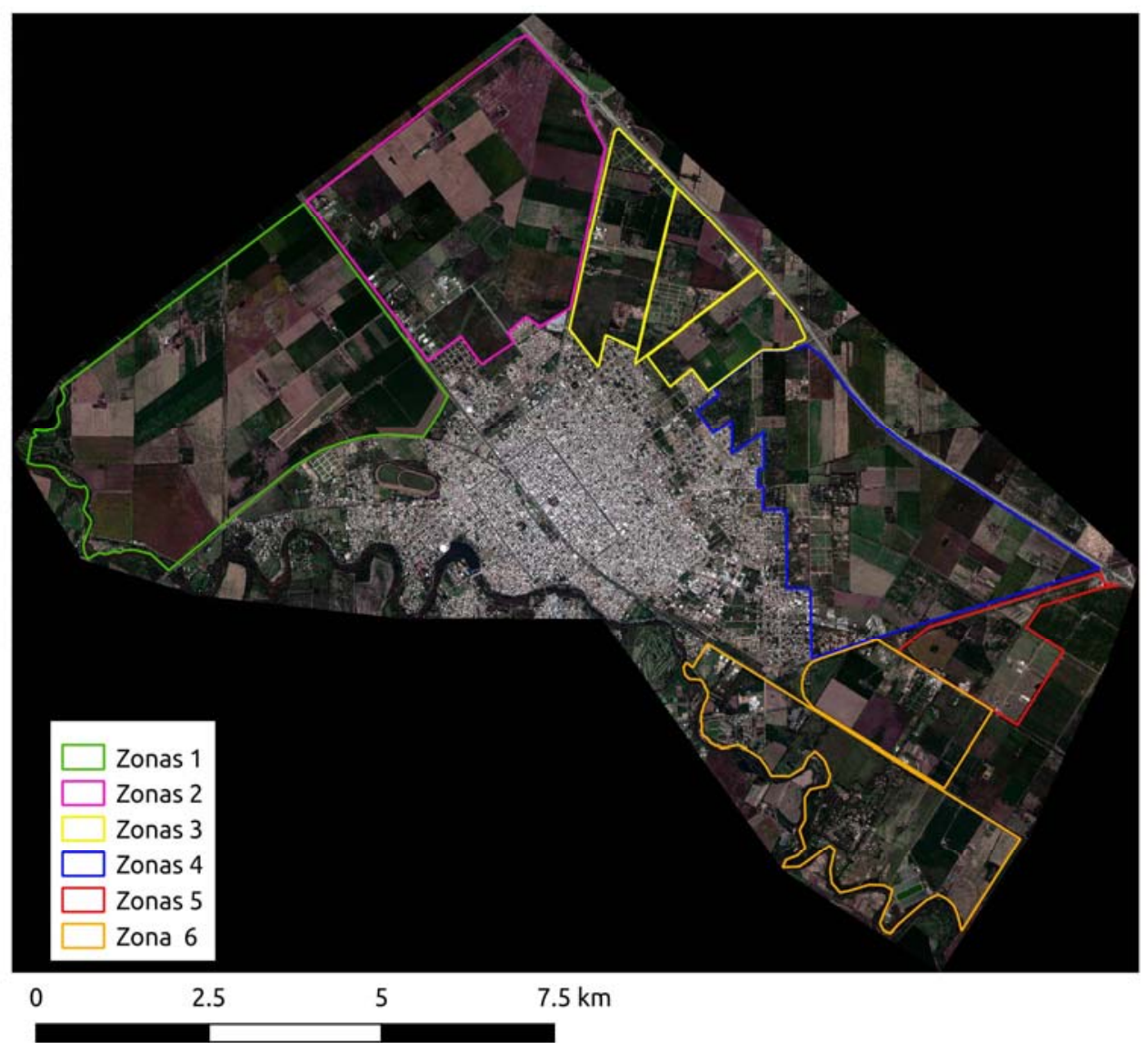

Figura 1. Zona Periurbana de Villa María, Córdoba, Argentina.

Publicado en formato digital: Ing. L. A. Guzmán, Dra. A Becker y Dr. J.C. Bedano. AVANCES Y ANÁLISIS EN LA CONSTRUCCIÓN DE INDICADORES DE PRESIÓN, ESTADO Y RESPUESTA PARA LA CALIDAD AMBIENTAL EN EL PERIURBANO DE VILLA MARÍA, CÓRDOBA, ARGENTINA. Revista Geográfica Digital. IGUNNE. Facultad de Humanidades. UNNE. Año 15. № 29. Enero - Junio 2018. ISSN 1668-5180 Resistencia, Chaco.

En: http://hum.unne.edu.ar/revistas/geoweb/default.htm 


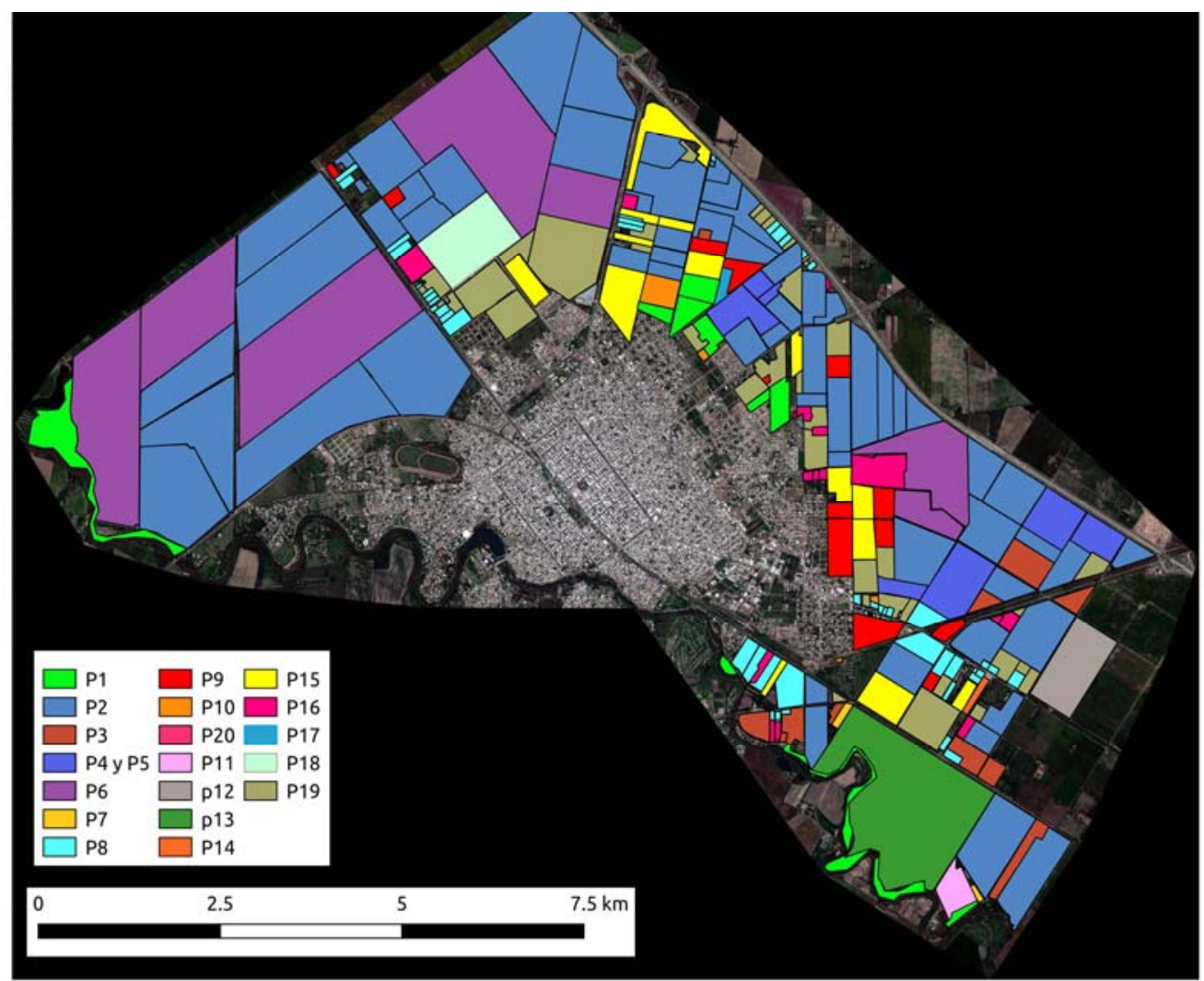

Figura 2: Parches de uso del suelo del periurbano de Villa María ${ }^{(2)}$

Se combinaron las fichas de caracterización descriptivas de Cantú et al. (2008) y la propuesta por Gaviño Novillo (1995).

Cantú et al. (2008) determinan las funciones de los indicadores en relación al concebir al ambiente por el grado de alcance de la calidad ambiental que se agrupa en:

Función Naturalidad: Da indicio del grado de naturalidad que puede aportar el indicador al análisis.

Función Fuente de Recursos: Determina si el indicador puede aportar a la extracción o uso de los recursos naturales materiales o de servicios.

Función Sumidero: La lectura del indicador aporta datos para analizar si el ambiente está siendo soporte de residuos, efluentes o desechos.

A esto se le sumó lo propuesto por Allen (2003) donde se analiza a que subsistema se aplica el indicador: natural, productivo o urbano. Esta incorporación es en función de que se visualicen los sistemas a los cuales se orientan e inferir indirectamente quienes están utilizando los servicios ambientales.

En relación a la condición particular que presenta el periurbano se construyeron indicadores propios que reflejen y aborden las dinámicas de transformación, la condición contextual con otros usos y los procesos de transformación. Estos indicadores responden a los conceptos planteados por la economía ecológica donde los usos y las actividades sociales son subsistemas de un gran sistema natural (Martínez Alier, 2004, 1998).

\section{Resultados}

De la combinación de las fichas para la construcción de indicadores se obtuvo una ficha que permitió tener mayor información respecto al indicador (Figura 3) contemplando los criterios establecidos por

Publicado en formato digital: Ing. L. A. Guzmán, Dra. A Becker y Dr. J.C. Bedano. AVANCES Y ANÁLISIS EN LA CONSTRUCCIÓN DE INDICADORES DE PRESIÓN, ESTADO Y RESPUESTA PARA LA CALIDAD AMBIENTAL EN EL PERIURBANO DE VILLA MARÍA, CÓRDOBA, ARGENTINA. Revista Geográfica Digital. IGUNNE. Facultad de Humanidades. UNNE. Año 15. N No 29. Enero - Junio 2018. ISSN 1668-5180 Resistencia, Chaco.

En: http://hum.unne.edu.ar/revistas/geoweb/default.htm 
Revista Geográfica Digital. IGUNNE. Facultad de Humanidades. UNNE. Año 15. № 29.

Enero - Junio 2018. ISSN 1668-5180 Resistencia, Chaco

la Comisión Económica para América Latina y el Caribe (CEPAL) de las Naciones Unidas en el trabajo de Quiroga Martínez (2009).

\begin{tabular}{|c|c|c|}
\hline \multicolumn{3}{|c|}{ Indicador de PRESIÓN/ ESTADO/ RESPUESTA } \\
\hline \multicolumn{2}{|l|}{ PARCHE O ZONA (a quien se aplica) } & $\begin{array}{l}\text { INDICADOR } \\
\text { (NOMBRE) }\end{array}$ \\
\hline \multirow{4}{*}{ INDICADOR } & Breve Definición & \\
\hline & Unidad de medida & \\
\hline & Formula de calculo & \\
\hline & Valor máximo y mínimo & \\
\hline \multirow{3}{*}{ SIGNIFICADO } & Finalidad/ Objetivo & \\
\hline & Relación con otros indicadores & \\
\hline & Fundamentación & \\
\hline \multirow{5}{*}{$\begin{array}{l}\text { EVALUACIÓN DE LA } \\
\text { DISPONIBILIDAD DE DATOS DE } \\
\text { FUENTES NACIONALES E } \\
\text { INTERNACIONALES }\end{array}$} & Datos necesarios para determinar el indicador & \\
\hline & Disponibilidad de datos & \\
\hline & Fuentes de datos & \\
\hline & Frecuencia de la medición & \\
\hline & Aplicación al pasado y al futuro & \\
\hline \multirow{3}{*}{$\begin{array}{l}\text { DESCRIPCIÓN DE LA } \\
\text { METODOLOGÍA Y DEFINICIONES } \\
\text { EN LAS QUE SE BASA }\end{array}$} & Definiciones y conceptos básicos & \\
\hline & Métodos de medición & \\
\hline & Limitaciones del indicador & \\
\hline
\end{tabular}

Figura 3: Ficha técnica para la conformación de los indicadores.

En función de los parches de uso de suelo en las 6 zonas del periurbano se obtuvieron 13 indicadores (Tabla 1): 6 de presión, 3 de estado y 4 de respuesta. Dichos indicadores responden las actividades agroecosistémicas, productivas, de estabilidad o inestabilidad de la actividad, condiciones naturales.

Publicado en formato digital: Ing. L. A. Guzmán, Dra. A Becker y Dr. J.C. Bedano. AVANCES Y ANÁLISIS EN LA CONSTRUCCIÓN DE INDICADORES DE PRESIÓN, ESTADO Y RESPUESTA PARA LA CALIDAD AMBIENTAL EN EL PERIURBANO DE VILLA MARÍA, CÓRDOBA, ARGENTINA. Revista Geográfica Digital. IGUNNE. Facultad de Humanidades. UNNE. Año 15. № 29. Enero - Junio 2018. ISSN 1668-5180 Resistencia, Chaco.

En: http://hum.unne.edu.ar/revistas/geoweb/default.htm 
Revista Geográfica Digital. IGUNNE. Facultad de Humanidades. UNNE. Año 15. № 29.

Enero - Junio 2018. ISSN 1668-5180 Resistencia, Chaco

\begin{tabular}{|c|c|c|c|}
\hline & Número del Indicador & Nombre del Indicador & Breve Definición \\
\hline & & & \\
\hline \multirow{6}{*}{ 嘆 } & I.PN ${ }^{\circ} 1$ (DC) & Densidad de caminos & $\begin{array}{l}\text { indicador indirecto de fraccionamiento de las zonas, perturbación y } \\
\text { accesibilidad a la producción. }\end{array}$ \\
\hline & I.PN² (CA) & $\begin{array}{l}\text { Ganadería extensiva- } \\
\text { Carga animal }\end{array}$ & $\begin{array}{l}\text { Es un indicador aplicable a los recursos suelo y agua e implica las } \\
\text { transformaciones que se operan sobre los mismos debido al tráfico y } \\
\text { permanencia de animales. Cuanto mayor es la carga , mayor será el grado de } \\
\text { afección del recurso y sus funciones de sumidero. }\end{array}$ \\
\hline & I.PN 3 (DUUD) & $\begin{array}{c}\text { Densidad de unidades } \\
\text { de uso dinámico }\end{array}$ & $\begin{array}{l}\text { Establece la relación dinámica del territorio con el uso. Mientras mayor sea el } \\
\text { numero de establecimientos dinámicos que existan por km², menor será la } \\
\text { presión que se ejerce sobre los recursos. }\end{array}$ \\
\hline & I.PN 4 (DP) & $\begin{array}{c}\text { Densidad de parches } \\
\text { por zona }\end{array}$ & $\begin{array}{l}\text { La diversidad productiva ponderada por la superficie nos da la diversidad que } \\
\text { ofrece esa zona en función de la superficie total del periurbano. }\end{array}$ \\
\hline & I.PN5 (DUUE) & $\begin{array}{c}\text { Densidad de unidades } \\
\text { de uso estático }\end{array}$ & $\begin{array}{l}\text { Establece la relación estática del territorio con el uso. Mientras mayor sea el } \\
\text { numero de establecimientos industriales, de vivienda, ladrilleras o sumidero } \\
\text { que existan por km², mayor será la presión que se ejerce sobre los recursos. }\end{array}$ \\
\hline & I.PN 6 (SE) & $\begin{array}{l}\text { Superficie de la } \\
\text { actividad extractiva }\end{array}$ & $\begin{array}{l}\text { La extracción de suelo para la construcción de ladrillos es una actividad que } \\
\text { modifica de forma permanente el suelo y el uso posterior del territorio. }\end{array}$ \\
\hline \multirow{3}{*}{ 窟 } & I.E.N ${ }^{\circ} 1$ (PI) & $\begin{array}{l}\text { Perímetro interno de } \\
\text { zona }\end{array}$ & $\begin{array}{l}\text { El perímetro interno de las zonas periurbana permite establecer la frontera de } \\
\text { contacto y de posible transformación. }\end{array}$ \\
\hline & I.E.N² (PZ) & $\begin{array}{c}\text { Cantidad de pozos de } \\
\text { extracción de agua }\end{array}$ & Permite estimar la situación en la que se encuentra el recurso agua subterránea. \\
\hline & I.E. $N^{\circ} 3(\mathrm{DA})$ & $\begin{array}{l}\text { Distribución de las } \\
\text { actividades }\end{array}$ & $\begin{array}{l}\text { La predominancia de cada actividad por zona y posteriormente en la superficie } \\
\text { total indica el peso de esa actividad en superficie ocupada total por ese tipo de } \\
\text { uso. }\end{array}$ \\
\hline \multirow{4}{*}{ 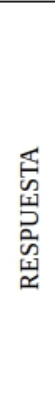 } & I.R.N ${ }^{\circ} 1$ (NCAOM) & $\begin{array}{l}\text { Número de campos } \\
\text { afectados por la OM } \\
\text { de resguardo } \\
\text { ambiental }\end{array}$ & Infiere la cantidad de productores o unidades productivas afectadas por la OM. \\
\hline & I.RN² (MCAOM) & $\begin{array}{l}\text { Metros afectados por } \\
\text { la OM de resguardo } \\
\text { ambiental }\end{array}$ & Superficie afectada por la OM. 6118 de resguardo ambiental. \\
\hline & I.RN³ (MVN) & $\begin{array}{l}\text { Metros² de vegetación } \\
\text { nativa }\end{array}$ & $\begin{array}{l}\text { Mayor la cantidad de } \mathrm{m}^{2} \text { de vegetación nativa es mayor la calidad ambiental y } \\
\text { los servicios ambientales que esta provee. }\end{array}$ \\
\hline & I.RN 4 (PVN) & $\begin{array}{l}\text { Perímetro de parche } \\
\text { con vegetación nativa }\end{array}$ & $\begin{array}{l}\text { La longitud del perímetro establece la superficie de contacto del parche con } \\
\text { otros parches, dando información sobre la posible penetración. }\end{array}$ \\
\hline
\end{tabular}

Tabla 1. Descripción de Indicadores.

Los Indicadores de Estado que se confeccionaron permiten visualizar la transformación y la predominancia de los usos del suelo.

En función de lo propuesto por Cantú et al. (2008) y Allen (2003) la categorización de los Indicadores se establece en la Tabla 2. Se definió la pertinencia que cada indicador cuenta con la clasificación de ambos autores, donde se respetó el origen de dichas clasificaciones.

Publicado en formato digital: Ing. L. A. Guzmán, Dra. A Becker y Dr. J.C. Bedano. AVANCES Y ANÁLISIS EN LA CONSTRUCCIÓN DE INDICADORES DE PRESIÓN, ESTADO Y RESPUESTA PARA LA CALIDAD AMBIENTAL EN EL PERIURBANO DE VILLA MARÍA, CÓRDOBA, ARGENTINA. Revista Geográfica Digital. IGUNNE. Facultad de Humanidades. UNNE. Año 15. № 29. Enero - Junio 2018. ISSN 1668-5180 Resistencia, Chaco.

En: http://hum.unne.edu.ar/revistas/geoweb/default.htm 
Revista Geográfica Digital. IGUNNE. Facultad de Humanidades. UNNE. Año 15. № 29. Enero - Junio 2018. ISSN 1668-5180 Resistencia, Chaco

Tabla 2. Fuente del indicador y subsistema al que aporta.

\begin{tabular}{|c|c|c|c|c|c|c|c|}
\hline \multirow[b]{2}{*}{ Número de indicador } & \multirow[b]{2}{*}{$\begin{array}{l}\text { Nombre del } \\
\text { Indicador }\end{array}$} & \multicolumn{3}{|c|}{ Fuente del indicador } & \multicolumn{3}{|c|}{ Subsistema al que aporta } \\
\hline & & Naturalidad & $\begin{array}{l}\text { Proveedor de } \\
\text { servicios }\end{array}$ & $\begin{array}{l}\text { Sumidero de } \\
\text { residuos }\end{array}$ & Natural & Productivo & Urbano \\
\hline I.PNº'1 (DC) & Densidad de caminos & $\mathrm{x}$ & & & $\mathrm{x}$ & $\mathrm{x}$ & \\
\hline I.PN² (CA) & $\begin{array}{c}\text { Ganadería extensiva- } \\
\text { Carga animal }\end{array}$ & & $\mathrm{x}$ & $\mathrm{x}$ & & $\mathrm{x}$ & \\
\hline I.PN³ (DUUD) & \begin{tabular}{|c|} 
Densidad de unidades \\
de uso dinámico
\end{tabular} & $\mathrm{x}$ & $\mathrm{x}$ & & $\mathrm{x}$ & $\mathrm{x}$ & \\
\hline I.PNº 4 (DP) & $\begin{array}{l}\text { Densidad de parches } \\
\text { por zona }\end{array}$ & $\mathrm{x}$ & $\mathrm{x}$ & $\mathrm{X}$ & $\mathrm{X}$ & $\mathrm{x}$ & $\mathrm{X}$ \\
\hline I.PN5 (DUUE) & $\begin{array}{c}\text { Densidad de unidades } \\
\text { de uso estático }\end{array}$ & & $\mathrm{x}$ & $\mathrm{x}$ & & $\mathrm{x}$ & $\mathrm{x}$ \\
\hline I.PNº 6 (SE) & $\begin{array}{c}\text { Superficie de la } \\
\text { actividad extractiva }\end{array}$ & & $\mathrm{x}$ & $\mathrm{x}$ & & & $\mathrm{x}$ \\
\hline I.E.Nº1 (PI) & $\begin{array}{l}\text { Perímetro interno de } \\
\text { zona }\end{array}$ & & & & & & \\
\hline I.E.N² (PZ) & $\begin{array}{c}\text { Cantidad de pozos de } \\
\text { extracción de agua }\end{array}$ & $\mathrm{x}$ & $\mathrm{X}$ & & & $\mathrm{x}$ & \\
\hline I.E.N³ (DA) & $\begin{array}{l}\text { Distribución de las } \\
\text { actividades }\end{array}$ & $\mathrm{x}$ & $\mathrm{x}$ & $\mathrm{x}$ & $\mathrm{x}$ & $\mathrm{x}$ & $\mathrm{x}$ \\
\hline I.R.N¹ 1 (NCAOM) & $\begin{array}{l}\text { Número de campos } \\
\text { afectados por la OM } \\
\text { de resguardo } \\
\text { ambiental }\end{array}$ & & & & & & \\
\hline I.RN² (MCAOM) & $\begin{array}{c}\text { Metros afectados por } \\
\text { la OM de resguardo } \\
\text { ambiental }\end{array}$ & & & & & & \\
\hline I.RN³ (MVN) & $\begin{array}{c}\text { Metros }^{2} \text { de vegetación } \\
\text { nativa }\end{array}$ & $\mathrm{x}$ & & & $\mathrm{x}$ & & \\
\hline I.RN (PVN) & $\begin{array}{l}\text { Perímetro de parche } \\
\text { con vegetación nativa }\end{array}$ & $\mathrm{x}$ & & & $\mathrm{x}$ & & \\
\hline
\end{tabular}

\section{Discusión}

De las funciones de naturalidad, proveedor de servicios y sumidero (Cantú et al.,2008) no son aplicables a todos los indicadores ya que esta clasificación fue hecha para el análisis de un recurso natural en específico como ser suelo o agua, cuando se intenta aplicar a indicadores más globales donde se intenta detectar una relación o una vulnerabilidad como puede ser el caso del Indicador de Estado (I.E.N ${ }^{\circ}$ : Perímetro interno de la zona). En este mismo sentido el Indicador de Respuesta (I.R.N ${ }^{\circ} 1$ y No2) relacionado a la Ordenanza Municipal (OM) de resguardo ambiental no responde a una fuente puntual de los propuestos.

Los indicadores de respuesta expuestos por Cantú et al. (2008) están relacionados principalmente, a normativas y condiciones políticas deseadas, dejando descubierta la búsqueda de la diversidad productiva, como así también la presencia de actividades de bajo impacto. Por ello algunos de los indicadores propuestos no se asocian a una fuente considerada por estos autores. Así también,

Publicado en formato digital: Ing. L. A. Guzmán, Dra. A Becker y Dr. J.C. Bedano. AVANCES Y ANÁLISIS EN LA CONSTRUCCIÓN DE INDICADORES DE PRESIÓN, ESTADO Y RESPUESTA PARA LA CALIDAD AMBIENTAL EN EL PERIURBANO DE VILLA MARÍA, CÓRDOBA, ARGENTINA. Revista Geográfica Digital. IGUNNE. Facultad de Humanidades. UNNE. Año 15. № 29. Enero - Junio 2018. ISSN 1668-5180 Resistencia, Chaco.

En: http://hum.unne.edu.ar/revistas/geoweb/default.htm 
Revista Geográfica Digital. IGUNNE. Facultad de Humanidades. UNNE. Año 15. № 29. Enero - Junio 2018. ISSN 1668-5180 Resistencia, Chaco

algunos indicadores no se asocian a los subsistemas propuestos por Allen (2003) el análisis de los subsistemas a los que aporta no puede ser establecido claramente para todos los indicadores ya que se trabaja con un espacio de transición entre lo urbano y rural.

Otros indicadores, por su parte, no responden a las clasificaciones de ambos autores, sin embargo, todos los indicadores cumplen con las condiciones mínimas que establece Quiroga Martínez (2009) en la serie de manuales de la Comisión Económica para América Latina y el Caribe (CEPAL), entendiendo las diferencias de escala.

Por lo expuesto, se puede establecer que algunos indicadores propuestos para el periurbano demuestran dinámicas de transformación y no la calidad de un recurso o un pertenecer a un subsistema puntual.

La conformación de los Indicadores de Respuesta $N^{0} 3$ y Nº aportan al análisis de los servicios ambientales que dan los ecosistemas naturales sin modificación o con mínima perturbación. Para estos se deben crear indicadores complementarios que permitan establecer la diversidad, riqueza y abundancia de dichos parches, ya que solo la medición de su superficie no refleja plenamente la importancia ecosistémica como fuera mencionado por González Zuarth et al (2014).

El catalogar los indicadores en los dos criterios propuestos permite visualizar el alcance de los mismos y que estos abarcan en su mayoría las condiciones del periurbano donde predominan los sistemas productivos y naturales, teniendo pequeñas inserciones de urbano. Así como también los grados de perturbación, quedando balanceados entre proveedor de servicio y naturalidad.

\section{Conclusiones}

La selección de Indicadores ambientales, replicables y sencillos, es una herramienta útil para la toma de decisiones en la gestión municipal de localidades medianas como Villa María, Córdoba, Argentina.

Una linea de base ambiental es impresindible para poder establecer una situación inicial y determinar la calidad ambiental en función a un referente y marcar el camino hacia un futuro deseado por medio de indicadores.

Los indicadores establecen criterios en función de los servicios ambientales y el análisis espacial dinámico para entender los movimientos y las relaciones de los parches y las zonas.

Es necesario confeccionar mayor cantidad de indicadores de estado y respuesta a fin de abordar los aspectos sociales y productivos del sector.

Finalmente, se puede establecer que el periurbano de Villa María es un espacio de transición entre la ciudad y el campo y que al contar con características de cada uno de estas unidades debe ser contemplado individualmente, ya que al aplicar indicadores exclusivamente urbanos o rurales se pierde de vista la esencia de la zona de transición. Sin embargo, se deben crear indicadores que conecten lo rural y lo urbano en este espacio abordando los procesos dinámicos de transformación y las exigencias a los recursos naturales.

\section{Bibliografía}

Allen, A. 2003. La interfase periurbana como escenario de cambio y acción hacia la sustentabilidad del desarrollo. Cuadernos del CENDES, Año 20, N53: 1-15.

Altieri, M.; Nicholls, C.I. 2000. Teoría y práctica para una agricultura sustentable. Serie Textos Básicos para la Formación Ambiental. PNUMA. Red de Formación Ambiental para América Latina y el Caribe.

Benlloch, P.I. 1993. Una propuesta metodológica para el estudio del paisaje integrado. Geographicalia, No30: 229:242.

Bosnero, H.; Pappalardo, J.; Sanabria J.; Carnero, M.; Bustos, V. 2006. Carta de Suelos de la Rep. Argentina, Hoja 3363-9, Villa María, Escala 1:50.000. Convenio Agencia Cba. Ambiente-INTA.

Publicado en formato digital: Ing. L. A. Guzmán, Dra. A Becker y Dr. J.C. Bedano. AVANCES Y ANÁLISIS EN LA CONSTRUCCIÓN DE INDICADORES DE PRESIÓN, ESTADO Y RESPUESTA PARA LA CALIDAD AMBIENTAL EN EL PERIURBANO DE VILLA MARÍA, CÓRDOBA, ARGENTINA. Revista Geográfica Digital. IGUNNE. Facultad de Humanidades. UNNE. Año 15. № 29. Enero - Junio 2018. ISSN 1668-5180 Resistencia, Chaco.

En: http://hum.unne.edu.ar/revistas/geoweb/default.htm 
Revista Geográfica Digital. IGUNNE. Facultad de Humanidades. UNNE. Año 15. № 29.

Enero - Junio 2018. ISSN 1668-5180 Resistencia, Chaco

Cabrera, A. 1971. Fitogeografía de la República Argentina. Boletín de la sociedad Argentina de Botánica. Vol XIV, N1-2.

Cantú, M.P.; Becker, A.R.; Bedano J.C. 2008. Evaluación de la sustentabilidad Ambiental en Sistemas Agropecuarios. Desarrollo y aplicación de la metodología del proyecto REDESAR (PICTR 439/03). Córdoba, Argentina. Fundación UNRC.

Carignano, C.; Kröhling, D., Degiovanni, S.; Cioccale, M. 2014. Geología de Superficie, Geomorfología. Relatorio del XIX Congreso Geológico Argentino: 747-821. Córdoba, Argentina (2-6 junio).

Cendrero Uceda, A. 1997. Indicadores de desarrollo sostenible para la toma de decisiones. Naturzale. N 12: 5-25.

Crojethovich, M. A. \& Barsky, A. 2012. Ecología de los bordes urbanos. En: Di Pace, M. \& Caride Bartrons, H. (Ed), Ecología Urbana: 185-232. Ed. UNGS. Argentina.

Di Pace, M. \& Caride Bartons, H. 2012. Ecología Urbana. Prometeo-UNGS. Buenos Aires.

Gaviño Novillo, M. 1995. Indicadores Ambientales y su aplicación. Cátedra UNESCO para el Desarrollado Sustentable. Foro latino Americano de Ciencias Ambientales: 1-44.

Gómez Orea, D.; Villarino, M.T.G. 2013. Evaluación de impacto ambiental. Mundi-Prensa Libros. España. 560 p.

Gonzalez Zuarth, C. A.; Vallarino, A.; Pérez Jiménez, J. C.; Low pfreng, A. 2014. Bioindicadores: Guardianes de nuestro futuro. Instituto Nacional de Ecología y Cambio Climático, D. F., México: 695-705.

Guzmán, L.A.; Castro, R.; Becker, A.; Furlan, M.L.; Rodríguez, J.M. 2016. Delimitación de parches de uso del suelo en la zona periurbana de Villa María, Córdoba, Argentina. Revista Mapping. Vol. 25 N177: 32-39.

Hernández-Jiménez, V.; Ocón, B.; Guillén, V. 2009. Espacios periurbanos. Transición de la ciudad al campo. Ecosostenible. N49: 5-12.

Instituto Nacional De Estadísticas y Censos (INDEC).Resultados del Censo 2010.(base de datos REDATAM) http://www.indec.gov.ar/bases-de-

datos.asp?solapa=5\&_ga=2.31973310.1876494495.1502482197-240676866.1495903759 (última

visita 2 de agosto de 2017)

Martínez Alier, J. 2004. Los conflictos ecológicos-distributivos y los indicadores de sustentabilidad. Revista Iberoamericana de Economía Ecológica (REVIBEC): 21-30.

Martínez Alier, J. 1998. Curso de Economía Ecológica. PNUMA. México D.F.:119-158.

Matteucci, S.D. 2008. Áreas Protegidas y el avance de las fronteras agrícolas y urbana. Fronteras. N7, año 7: 1-10.

Matteucci, S.D. 1998. La creciente importancia de los estudios del medio ambiente. En Matteucci SD y Buzai G (compiladores), Sistemas ambientales Complejos: herramientas de análisis espacial. Ed. Eudeba. Buenos Aires: 35-62.

Muñoz, F. 2010. Revitalización versus rurbanización. Estrategias de política territorial en Catalunya. En Bertuzzi, M. L. (comp.) Vivir en el paisaje. Reflexiones sobre problemática urbana de la costa: 26-39. Ed. Ediciones UNL. Santa Fe.

OECD. 2008. OECD. Key Environmental Indicators https://www.oecd.org/general/searchresults/?q=environmental\%20indicators\&cx=01243260174851139 1518:xzeadub0b0a\&cof=FORID:11\&ie=UTF-8 (última visita 05 de enero de 2017).

Quiroga Martínez, R. 2009. Guía metodológica para desarrollar indicadores ambientales y de desarrollo sostenible en países de América Latina y el Caribe. CEPAL. Serie manuales 61. Santiago de Chile. Chile: 58-89.

Seiler, R.A.; Vianco, A.M. 2014. Metodología para generar indicadores de sustentabilidad de sistemas productivos: Región Centro Oeste de Argentina. Río Cuarto, Argentina. Ed. UniRío: 1545.

Zulaica, M. L., Ferraro, R. 2012. Procesos de crecimiento, indicadores de sustentabilidad urbana y lineamientos de intervención en el periurbano marplatense. ARQUISUR, 1(2): 124-142.

Zulaica, M.L. 2010. Transformaciones territoriales en el sector sur del periurbano marplatense: Causas y consecuencias ambientales. Tesis Doctoral. Universidad Nacional del Sur. Bahía Blanca. $325 \mathrm{p}$.

Publicado en formato digital: Ing. L. A. Guzmán, Dra. A Becker y Dr. J.C. Bedano. AVANCES Y ANÁLISIS EN LA CONSTRUCCIÓN DE INDICADORES DE PRESIÓN, ESTADO Y RESPUESTA PARA LA CALIDAD AMBIENTAL EN EL PERIURBANO DE VILLA MARÍA, CÓRDOBA, ARGENTINA. Revista Geográfica Digital. IGUNNE. Facultad de Humanidades. UNNE. Año 15. № 29. Enero - Junio 2018. ISSN 1668-5180 Resistencia, Chaco.

En: http://hum.unne.edu.ar/revistas/geoweb/default.htm 


\section{Nota al pie}

(1) Parche de uso de suelo: Basados en Matteucci (2008) se entiende como parche a una unidad homogénea donde predomina un tipo de actividad o condición particular (Muñoz, 2010). Los parches pueden variar en su forma, superficie y entorno, pueden ser de igual denominación, colindantes y diferenciarse por su gestión o pertenencia.

(2)P1:Relicto de espinal. Suelo destinado a la conservación de espacio natural o que cuentan con ejemplares de especies nativas y/o renovales.

P2: Agrícola extensiva. Cultivos extensivos, caracterizada por tener principalmente siembra de soja y maíz.

P3: Agrícola intensiva. Horticultura, fruticultura. Plantaciones diversas y eventualmente con invernáculos.

P4: Agrícola-ganadero. Campos de producción agrícola con introducción de animales.

P5: Ganadería. Cría, recría y engorde de bovinos.

P6: Tambo. Cría de bovinos para la producción de leche.

P7: Producción intensiva animal. Pollo parrillero, conejos y/o cerdos.

P8: Industrial. Instalaciones industriales mixtas distribuidas fuera del Parque Industrial.

P9: Extractivo. Extracción de suelo para producción de ladrillos o que muestran rastros de haber albergado la actividad.

P10: Basurales clandestinos. Terrenos utilizados para el depósito de Residuos de diferentes tipos, sin autorización municipal para esta la actividad.

P11: Planta de Tratamiento de Efluentes Líquidos. Espacio utilizado para tratamiento de efluentes cloacales. P12: Parque Industrial y Tecnológico Villa María. Predio establecido por OM (Ordenanza Municipal) 5.907 para la localización de emprendimientos Industriales.

P13: Fabricaciones Militares Villa María. Fábrica de pólvoras perteneciente a Fabricaciones Militares.

P14: Villa Albertina. Barrio establecido en zona industrial censado en el 2010 aparte de Villa María.

P15: Loteos recientes en la zona periurbana. Loteos establecidos fuera de la continuidad urbana con presencia parcial o no de viviendas.

P16: Viviendas y recreación. Casa quintas y predios de recreación.

P17: Escuela Granja Los Amigos. Espacio de uso mixto con fines educativos con uso de horticultura y ganadería a pequeña escala.

P18: Universidad Nacional de Villa María. Campus de la Universidad Nacional de Villa María, cuenta con campo agrícola experimental, campo recreativo, módulos de educación, residencias y pequeño relicto de bosque nativo. P19: Campos sin actividad. Antiguos campos productivos agrícolas sin actividad reciente con presencia de vegetación.

Publicado en formato digital: Ing. L. A. Guzmán, Dra. A Becker y Dr. J.C. Bedano. AVANCES Y ANÁLISIS EN LA CONSTRUCCIÓN DE INDICADORES DE PRESIÓN, ESTADO Y RESPUESTA PARA LA CALIDAD AMBIENTAL EN EL PERIURBANO DE VILLA MARÍA, CÓRDOBA, ARGENTINA. Revista Geográfica Digital. IGUNNE. Facultad de Humanidades. UNNE. Año 15. N No 29. Enero - Junio 2018. ISSN 1668-5180 Resistencia, Chaco.

En: http://hum.unne.edu.ar/revistas/geoweb/default.htm 